\title{
Study of Pressure Sensors Placement using an Abdominal Aortic Aneurysm (AAA) Model
}

\author{
L.A. Rocha, A. Sepulveda, A.J. Pontes \& J.C. Viana \\ Institute for Polymers and Composites/I3N, University of Minho \\ PORTUGAL
}

Isa C. T. Santos \& João Manuel R. S. Tavares

Instituto de Engenharia Mecânica e Gestão Industrial / Faculdade de Engenharia, Universidade do Porto

PORTUGAL

ABSTRACT: An Abdominal Aortic Aneurysm (AAA) model for post-EVAR (endovascular aneurysm repair) analysis, including the blood flow, the bifurcated stent-graft, the aorta aneurysm wall motion and the stagnant blood inside the aneurysm sac, was built and solved using a Fluid Structure Interaction (FSI) code. The post-EVAR analysis aims to check the feasibility of EVAR surveillance using a remote pressure sensor, and the study of the pressure variations inside the aneurysm sac to determine the best placement position for the pressure sensor(s). First results suggest that aneurysm sac pressure measurement is feasible and can be a good indicator of aneurysms post-EVAR evolution.

Keywords: AAA Model, CFD, Aneurysm pressure sensing

\section{INTRODUCTION}

\subsection{Abdominal aortic aneurysms (AAA)}

An aneurysm can be defined as a permanent and irreversible localized dilatation of an artery, having at least a $50 \%$ increase in diameter compared with the healthy one. It can appear anywhere, but it occurs most commonly in the aorta, as well as in arteries located at the base of the brain and in the legs.

Two treatments are currently available for the treatment of aneurysms: conventional surgical repair (open surgery) (Myers et al. 2001) and endovascular aneurysm repair (EVAR) (Parodi et al. 1991). The first involves the replacement of the damaged section of the aorta with a prosthetic graft through a surgical procedure (a large incision in the abdomen). EVAR is a minimally invasive procedure in which a stent-graft is guided from the femoral artery to the affected artery segment in order to prevent wall rupture shielding the aneurysm from the blood pressure. The latter is usually associated with less physiological derangement, lower morbidity and mortality, and more rapid recovery than open surgery (Chuter et al. 2004) but, after the procedure, regular surveillance to detect and prevent complications such as graft migration, stent fracture, endoleaks, enlargement of the aneurysm sac, and AAA rupture is required.

Comparing both treatments, EVAR is preferable due to the fact of being less stressful and reducing significantly systemic complications (Rutherford \& Krupski 2004), as well as having lower costs of inpatient stay and less or no need for intensive care facilities during recovery. The durability of open surgery, established with long-term follow-up studies, is excellent (Rutherford \& Krupski, 2004), so good that there is little or no requirement for long-term surveillance, in contrast with EVAR whose current results suggest that there is a need for increased surveillance and re-intervention (Hayter et al. 2005). Considering the longer life expectancies and the rising public expectations for quality of life, the costs associated to the follow-up can jeopardize EVAR's effectiveness.

\subsection{EVAR Surveillance}

The current surveillance protocol involves imaging at 1,6 , and 12 months after the procedure, and thereafter, on an annual basis. In order to reduce and even eliminate these exams, new surveillance technologies are being investigated and the most promising technique identified so far is remote pressure sensing (Springer et al. 2007). Up to now, existing sensors are randomly placed on the aneurysm sac and only provide information, namely the pressure, regarding a single point.

Several studies using full AAA models (stentgraft, aorta and aneurysm sac) have been presented in the literature (Li \& Kleinstreuer, 2006, Frauenfelder et al. 2006, Scotti \& Finol, 2007), but they mainly studied the drag forces on the stent-graft, or the stresses on the vessel that can lead to rupture.

This paper introduces a Computational Fluidic Model (CFD) with Fluid Structure Interaction (FSI) of an AAA to study the pressure variations inside the aneurysm sac after stent-graft placement. The goal is to determine if there is a best location for placement of pressure sensors and to check if more information (besides the aneurysm sac pressure) can be retrieved. Additionally, the influence of AAA geometry on the sac pressure is also analyzed. 


\section{AAA MODEL}

\subsection{Geometry}

A full parametric script was implemented in a CAD software for the 3D modeling of the AAA geometry (Fig. 1). The geometry of the AAA is based on typical fusiform aneurysms geometries, including models built from CT-scan available in literature ( $\mathrm{Li} \&$ Kleinstreuer, 2006, Vorp, 2007).

a)

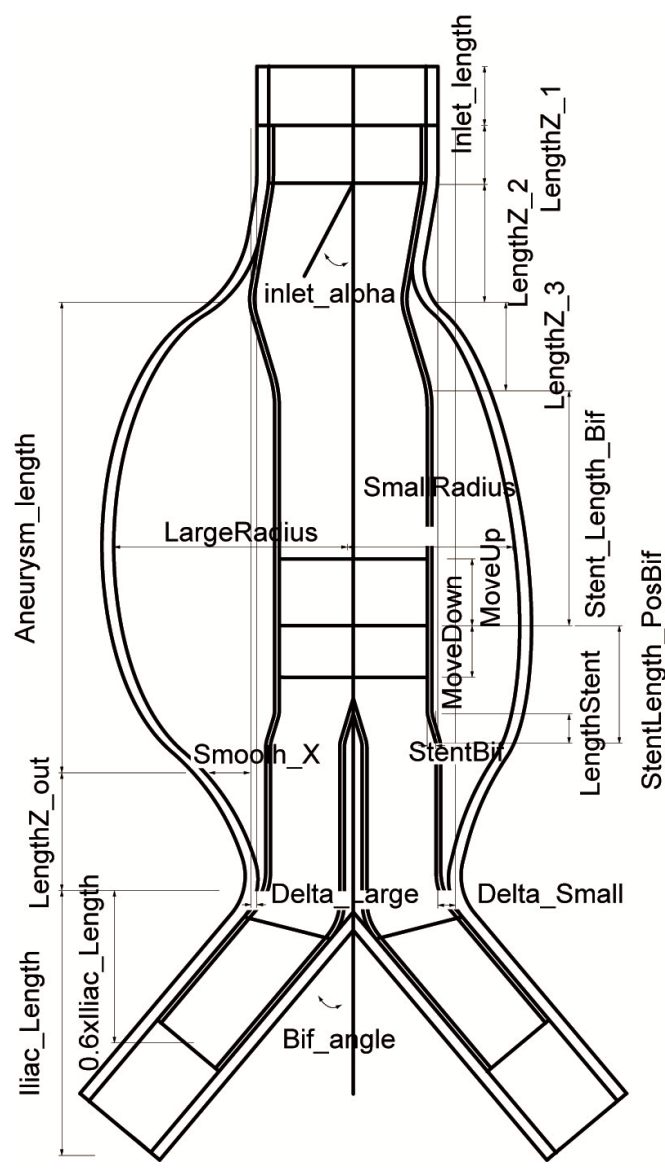

b)

c)
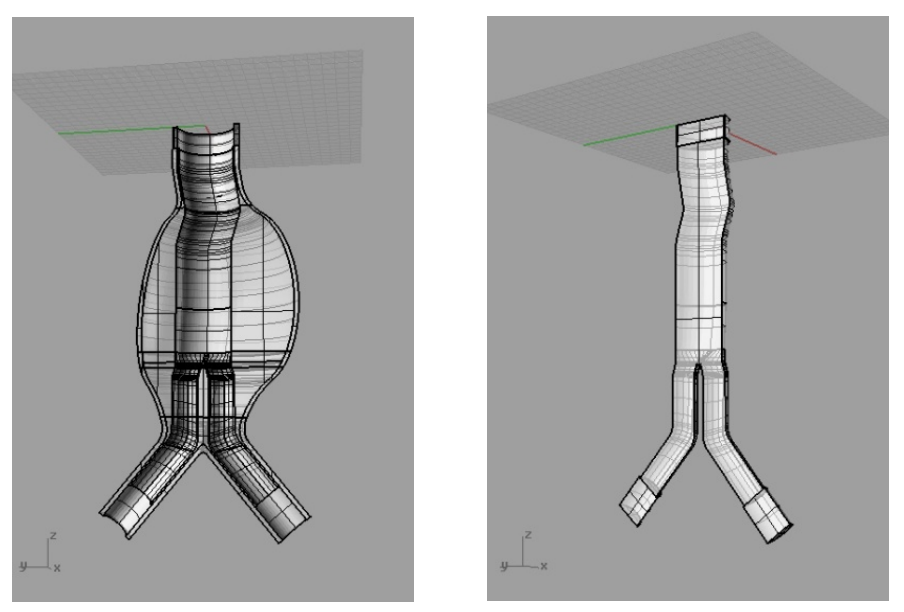

Figure 1. AAA geometry: a) main modeling parameters, b) 3D mechanical model and c) 3D fluidic model.

For the study presented here, aorta radius and aorta wall thicknesses of $2.5 \mathrm{~cm}$ and $2.5 \mathrm{~mm}$ respec- tively, were used. The aneurysm has a length of 10.5 $\mathrm{cm}$ and a main radius of $6.7 \mathrm{~cm}$.

\subsection{Material Properties}

The transient simulation used considers the pulsate nature of blood flow. The model includes the blood flow, the bifurcated stent-graft, the aorta wall motion (including the aneurysm wall) and the stagnant blood inside the aneurysm sac (essential for the pressure simulation inside the sac).

a)

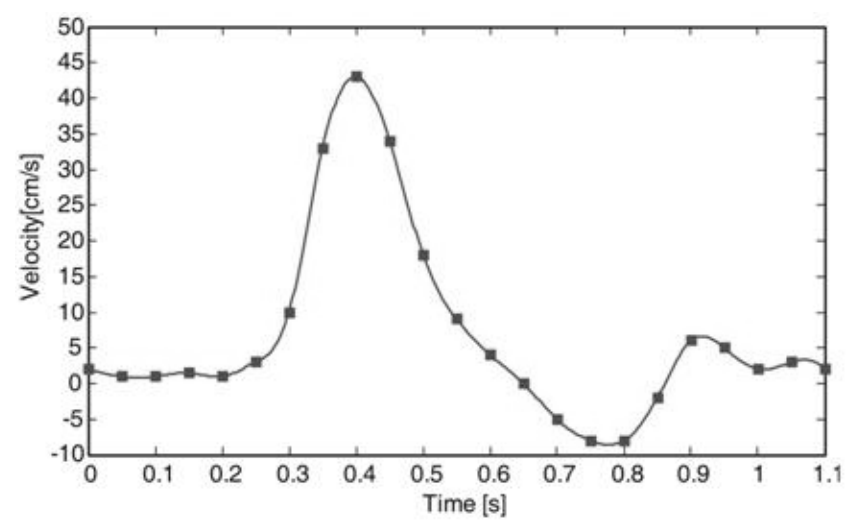

b)

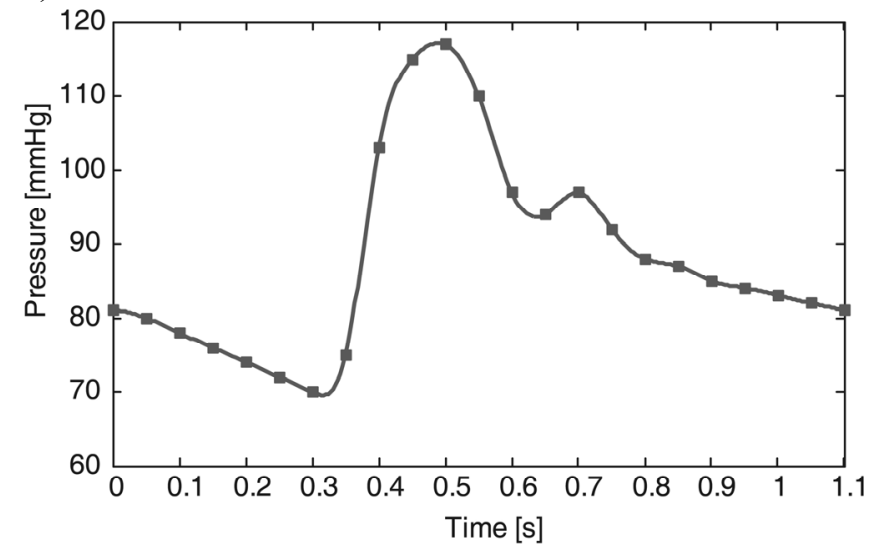

Figure 2. Fluidic boundary conditions: a) inlet velocity and b) outlet pressure.

The ANSYS multiple code coupling (MFX) with FSI coupling between Ansys and CFX was used to solve the model. Newtonian, laminar and incompressible blood flow was assumed with a density of $\rho=1.05 \mathrm{~g} / \mathrm{cm}^{3}$ and a viscosity of $v=0.0035$ Pa.s.

The diseased AAA wall was modeled as a nonlinear, isotropic, elastic material with a density $\rho=$ $1.2 \mathrm{~g} / \mathrm{cm}^{3}$, a Young's Modulus $\mathrm{E}=4.6 \mathrm{MPa}$ and a Poisson's ratio of 0.49 ( $\mathrm{Li} \&$ Kleinstreuer, 2006). The healthy part of the aorta (AAA neck) and iliac were also assumed to be a non-linear, isotropic, elastic material with a density $\rho=1.2 \mathrm{~g} / \mathrm{cm}^{3}$, a Young's Modulus E $=2 \mathrm{MPa}$ and a Poisson's ratio of 0.45 . The stent graft-graft was modeled with SHELL elements, and was assumed to be a non-linear, isotropic material with a Young's Modulus E $=10 \mathrm{MPa}$ and a Poisson's ratio of 0.3 .

The aneurysm sac, an important part of the model for the pressure analysis, was modeled as a stagnant 
liquid by using FLUID80 element from the ANSYS element library. This element allows the simulation of stagnant fluids in containers with no flow.

\subsection{Boundary Conditions}

The mechanical domain of the simulation was assumed to have zero displacement at the top of the AAA neck and at the bottom of the iliacs, while a time dependent uniform velocity was applied at the inlet of the fluidic domain (Fig. 2a) and a time dependent normal traction (due to luminal pressure) on the outlet (Fig. 2b). A full cardiac cycle (1.1 s) was simulated.

\section{RESULTS AND DISCUSSION}

\subsection{Simulation Results}

Initially, a simpler AAA model without stent-graft was used to validate the FSI solver. These initial simulation results showed a maximum aorta displacement of $1.9 \mathrm{~mm}$ and a maximum stress (von Mises) around $0.4 \mathrm{MPa}$ in good agreement with the data from Li \& Kleinstreuer, 2006 (using a similar geometry size).

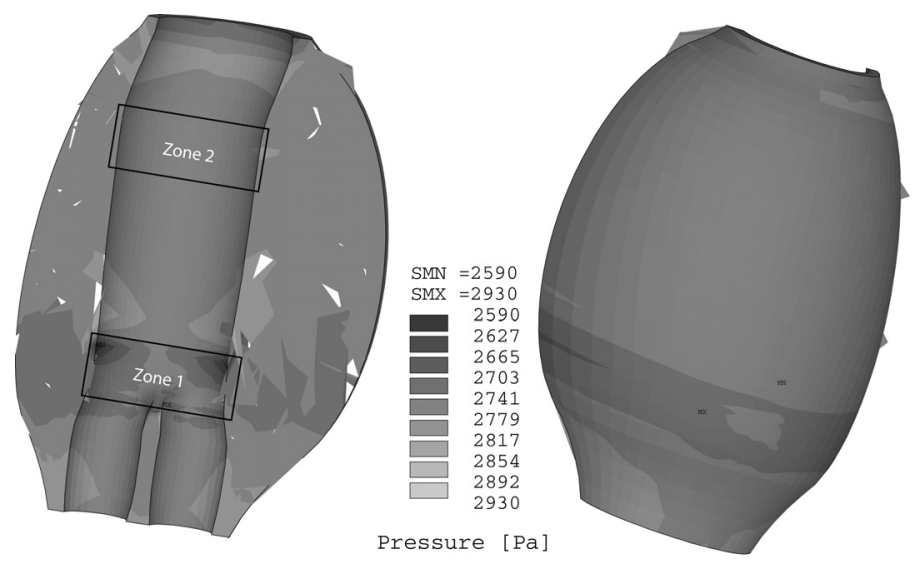

Figure 3. Results of the aneurysm sac pressure during systolic pressure $(\mathrm{t}=0.5 \mathrm{~s})$.

Next, the full AAA model with stent-graft was simulated and Figure 3 shows the pressure inside the aneurysm sac at systolic pressure $(\mathrm{t}=0.5 \mathrm{~s})$. Clearly, some pressure variations along the aneurysm sac are visible with the minimum pressure occurring close to the stent-graft bifurcation. A closer look to the stent-graft displacement at the same simulation time (Fig. 3) suggests that the minimum pressures are related to the maximum stent-graft displacements.

In order to assess the pressure variations inside the aneurysm sac during one cardiac cycle, two zones were defined (zone 1 and 2 in Fig. 3 ) and the mean pressures within those regions were plotted along with the pressure boundary condition at the outlet. The results are depicted in Figure 4.

\subsection{Discussion}

These initial simulations imply that the pressure variations within the aneurysm sac are related to the displacement of the stent-graft caused by the luminal pressure. If this is the case, the placement of one sensor on the region with less structural stability (higher displacement) might be a good indicator, when compared to other sensor placed elsewhere within the aneurysm sac, of the structural integrity of the stent-graft.

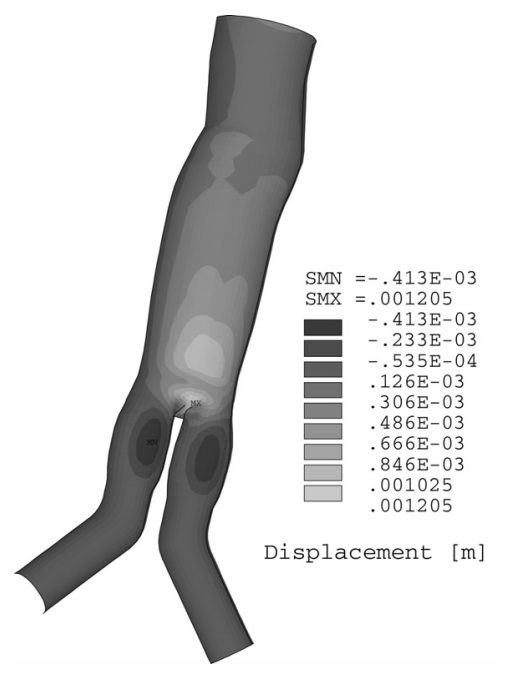

Figure 4. Stent-graft displacement during systolic pressure $(\mathrm{t}=$ $0.5 \mathrm{~s})$.

Since the pressure variations inside the aneurysm sac seem to be related to the stent-graft material (structural behavior), a second set of simulations were performed using a higher Young's Modulus for the stent-graft material, $\mathrm{E}=60 \mathrm{MPa}$.

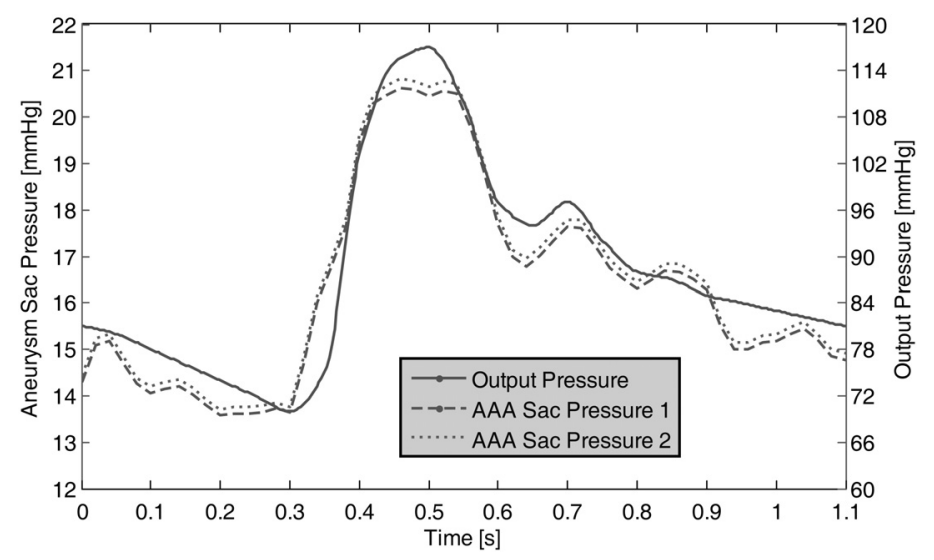

Figure 5. Pressures inside aneurysm sac (zone 1 and 2 in Fig. 3 ) and pressure boundary condition at the outlet.

The second set of simulations (Fig. 6) clearly shows that the pressure within the aneurysm sac depends on the structural behavior of the stent-graft material. In fact, simulations reveal that a large drop on the pressure within the aneurysm $(>10 \mathrm{mmHg}$ ) sac occurs when the Young's Modulus is increased from $10 \mathrm{MPa}$ to $60 \mathrm{MPa}$. As in the previous simulation, the minimum pressures within the aneurysm 
sac occur around the area where the stent-graft displacement is higher (Fig. 6b).

a)

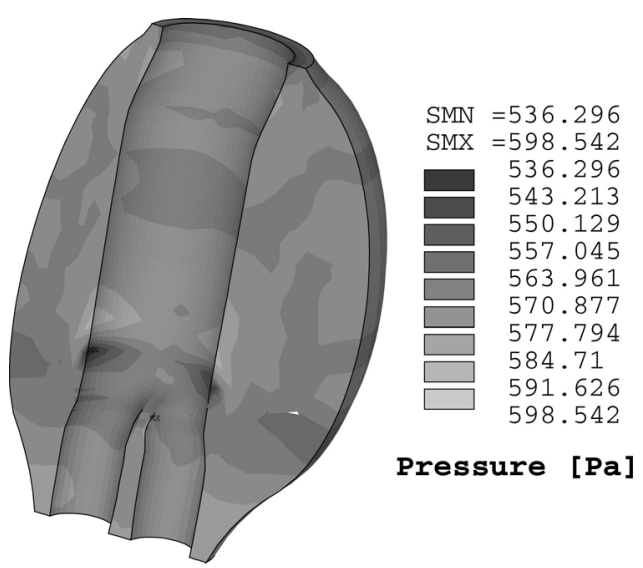

b)

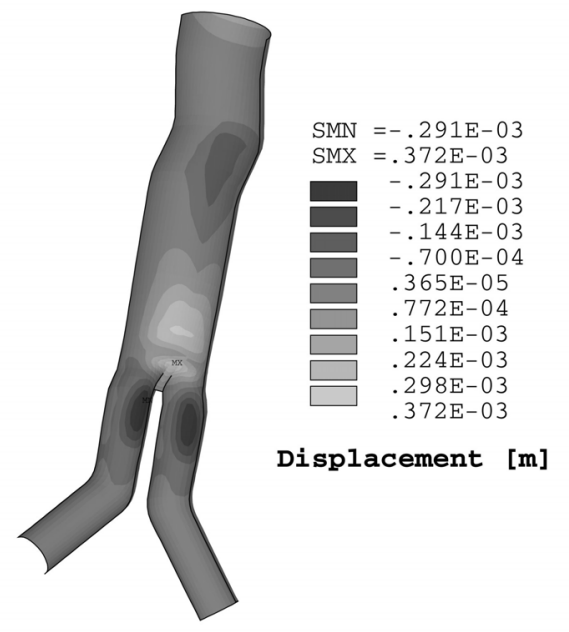

Figure 6. AAA simulations using a Young's Modulus of 60 MPa for the stent-graft material: a) aneurysm sac pressure and b) stent-graft displacement during systole $(t=0.5 \mathrm{~s})$.

Figure 7 compares the aneurysm sac pressure on zone 1 (Fig. 3) during the total cardiac cycle for the two simulated models. In both cases, the aneurysm sac transient pressure changes are related to the luminal pressure.

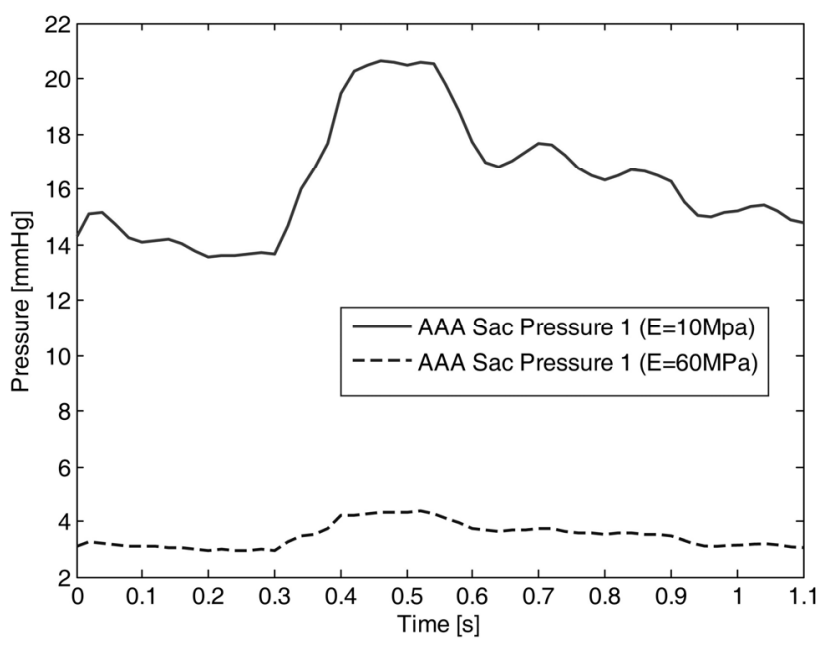

Figure 7. Pressures inside aneurysm sac on zone 1 (Fig. 3) for different stent-graft material properties.

\section{CONCLUSIONS}

An abdominal aortic aneurysm CFD model with FSI was developed to study the suitability of using pressure sensors to detect post-EVAR complications. The results demonstrate that pressure sensing in the aneurysm sac can be used both for leakage detection and to measure systolic and diastole blood pressures and indicate that the pressure within the aneurysm sac depends on the stent-graft material structural behavior.

Although more simulations are required to evaluate the best location for the placement of a cluster of pressure sensors, the results obtained suggest that the differences in pressure within the aneurysm sac can be an indicator of the stent-graft material integrity.

\section{ACKNOWLEDGEMENTS}

This work is supported by FCT under the project MIT-Pt/EDAM-EMD/0007/2008.

\section{REFERENCES}

Chuter, T., Parodi, J. C. and Lawrence-Brown, M. 2004. Management of abdominal aortic aneurysm: a decade of progress. Journal of Endovascular Therapy, 11(Sup. II): S82-S95.

Frauenfelder, T., Lotfey, M., Boehm, T., \& Wildermuth, S. 2006. Computational fluid dynamics: hemodynamic changes in abdominal aortic aneurysm after stent-graft implantation. Cardiovascular and interventional radiology, 29(4), 613-623.

Hayter C.L., Bradshaw S.R., Allen R.J., Guduguntla M. \& Hardman D.T. 2005. Follow-up costs increase the cost disparity between endovascular and open abdominal aortic aneurysm repair. Journal of Vascular Surgery, 42(5), 912918.

Li, Z., \& Kleinstreuer, C. 2006. Analysis of biomechanical factors affecting stent-graft migration in an abdominal aortic aneurysm model. J. Biomechanics, 39(12), 2264-2273.

Myers, K., Devine, T., Barras, C. \& Self, G. 2001. Endoluminal Versus Open repair for abdominal aortic aneurysms. $2^{\text {nd }}$ Virtual Congress of Cardiology.

Parodi, J.C., Palmaz, J.C. \& Barone, H.D. 1991. Transfemoral intraluminal graft implantation for abdominal aortic aneurysms. Annals of Vascular Surgery, 5(6), 491-499.

Rutherford, R.B. \& Krupski, W.C. 2004. Current status of open versus endovascular stent-graft repair of abdominal aortic aneurysm. Journal of Vascular Surgery, 39(5), 11291139.

Scotti, C., \& Finol, E. 2007. Compliant biomechanics of abdominal aortic aneurysms: A fluid-structure interaction study. Computers \& Structures, 85(11-14), 1097-1113.

Springer, F., Günther, R.W. \& Schmitz-Rode T. 2007, Aneurysm sac pressure measurement with minimally invasive implantable pressure sensors: An alternative to current surveillance regimes after EVAR?. CardioVascular and Interventional Radiology. 31(3): p. 460-467.

Vorp, D. A. 2007. Biomechanics of abdominal aortic aneurysm. J. Biomechanics, 40(9), 1887-902. 